\title{
Transurethral Drainage of Prostatic Abscess Using 120 W Holmium Laser Technology
}

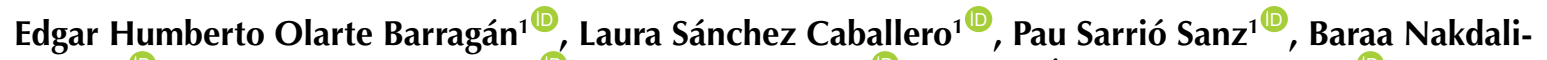 \\ Kassab $^{1}$, Miguel Gómez Garberí1 ${ }^{\mathbb{D}}$, Luis Gómez Pérez ${ }^{(\mathbb{D}}$, Manuel Ángel Ortiz Gorraiz ${ }^{(\mathbb{D}}$ \\ 'Department of Urology, Hospital Universitario San Juan de Alicante, Alicante, Spain
}

*Correspondence to Laura Sánchez Caballero, Email: laurasanchezc10@gmail.com

Received: February 19, 2021 Accepted: July 19, 2021

Published online 25 December, 2021

\section{Introduction}

Prostatic abscess as a complication of urinary tract infection is a rare but potentially serious pathology in the context of bacterial prostatitis. ${ }^{1}$ In terms of etiology, the microorganism found most frequently to be the cause is Escherichia coli in $60 \%-80 \%$ of cases. $^{2}$ Other important pathogens are Pseudomonas species, Staphylococcus and occasionally anaerobic bacteria. ${ }^{3}$ The prevalence of prostatic abscesses caused by Klebsiella pneumoniae varies across geographic areas. K. pneumoniae is the main cause in southern Taiwan ${ }^{3}$ and the second most frequent cause after E. coli in South Korea. ${ }^{4}$

Due to the high mortality rate, which ranges from 3\%$16 \%,{ }^{3}$ early treatment is necessary. This should include parenteral or oral administration of antibiotics that are sometimes associated with surgical drainage of the abscess to reduce severe complications. ${ }^{5}$

We present the case of a 72-year-old male who presented a prostatic abscess secondary to K. pneumoniae with concomitant meningitis caused by the same microorganism, with the patient requiring urgent surgical drainage.

\section{Case Report}

The patient was a 72-year-old man with a history of dilated cardiomyopathy of uncertain origin with a ventricular ejection fraction of $25 \%$. The patient had a history of urological treatment and a permanent urinary catheter for acute urinary retention episodes, with a prostate volume of $167 \mathrm{~g}$. He came to the emergency department suffering from asthenia, malaise, dyspnea and fever of up to $40^{\circ} \mathrm{C}$. His heart rate was 115 beats per minute. The analysis showed elevated acute phase reactants with a C-reactive protein of $20.37 \mathrm{mg} / \mathrm{dL}(0-0.5 \mathrm{mg} / \mathrm{dL})$, procalcitonin of $1.08 \mathrm{ng} / \mathrm{mL}(0-0.5 \mathrm{ng} / \mathrm{mL}), 21.4 \times 109 / \mathrm{L}$ leukocytes $(4-10 \times 109 / \mathrm{L})$ with $93 \%$ neutrophilia, hemoglobin of $12.3 \mathrm{~g} / \mathrm{dL}(14-17 \mathrm{~g} / \mathrm{dL})$, and $160109 / \mathrm{L}$ platelets (140$45109 / \mathrm{L})$. Prothrombin time was 17 seconds (9.40-12.50 seconds) and Quick coagulation test was 65\% (70\%$100 \%)$. The patient was admitted to internal medicine with a diagnosis of sepsis probably due to an infection of the urinary tract. Empirical treatment was started with intravenous Meropenem. During his hospitalization, he presented clinical worsening with nuchal rigidity and a decreased level of consciousness. A whole body computed tomography (CT) scan was requested. The cranial CT revealed the presence of leptomeningeal enhancement, compatible with meningitis. In addition, pelvic CT showed a liquid collection of $3 \times 5 \mathrm{~cm}$, probably abscessed in the right prostatic lobe (Figure 1).

A lumbar puncture was performed and the cerebrospinal fluid (CSF) obtained showed unclear characteristics and biochemistry compatible with meningitis of bacterial etiology. The culture of CSF was positive for multisensitive $K$. pneumoniae as well as the blood cultures. In view of the poor clinical evolution, the patient was admitted to the intensive care unit (ICU). Therefore, as he was a hemodynamically unstable patient in the context of

Please cite this article as follows: Olarte Barragán EH, Sánchez Caballero L, Sarrió Sanz P, Nakdali-Kassab B, Gómez Garberí M, Gómez Pérez L, et al. Transurethral drainage of prostatic abscess using 120 w holmium laser technology. J Lasers Med Sci. 2021;12:e80. doi:10.34172/jlms.2021.80. 


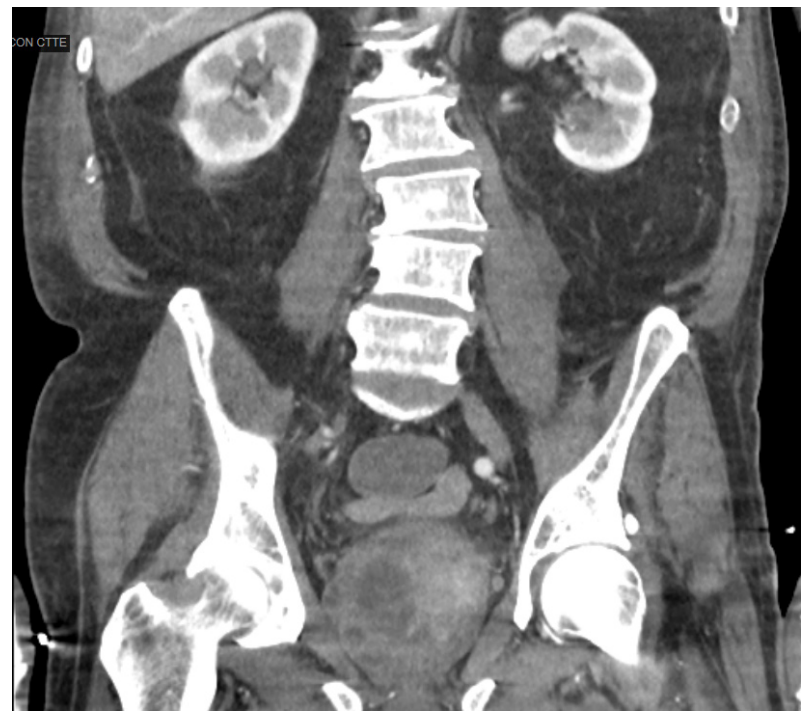

Figure 1. Right lobe prostatic abscess.

bacterial meningitis and prostatic abscess, the decision was taken to perform an urgent surgical intervention by drainage of the prostatic collection.

A cystoscopy was carried out, revealing an enlarged prostate, especially affecting the right prostatic lobe, which concurred with the preoperative CT scan. The decision was decided to carry out transurethral drainage by means of a $120 \mathrm{~W}$ holmium laser with MOSES technology, using 550 micron fiber. The incision was started at the 7 oclock position of the cystoscopic schedule at the level of the bladder neck and ended at the level of the verumontanum, with an energy of $1.2 \mathrm{~J}$, a frequency of $20 \mathrm{~Hz}$ and a total power of $24 \mathrm{~W}$ (Figure 2). The incision was deepened to the opening of the abscess cavity for drainage (Figure 3). A sample was taken for culture, and during the procedure, all the purulent material was eliminated by continuous irrigation with saline solution, and the cavity was completely cleaned. Hemostasis was precision-controlled, with the laser device set at $50 \mathrm{~Hz}-1 \mathrm{~J}(20 \mathrm{~W})$. A 22 French three-way urinary catheter was inserted. The culture of the purulent material was positive for a multi-sensitive strain of $K$. pneumoniae. The postoperative course was uneventful. After the operation, the patient showed clinical and analytical improvement and was discharged from the ICU two days after the intervention and from the hospital ward after 20 days.

\section{Discussion}

Prostatic abscesses are a rare clinical entity that represents $0.5 \%$ to $2 \%$ of all prostatic diseases and they are potentially serious, with a mortality rate of 6\%-30\%. ${ }^{6}$ Their etiopathogenesis is due to retrograde flow of contaminated urine, as well as the presence of prostatitis of torpid evolution or through hematogenous dissemination of another infectious focus. ${ }^{7}$ The most important predisposing factors are diabetes mellitus,

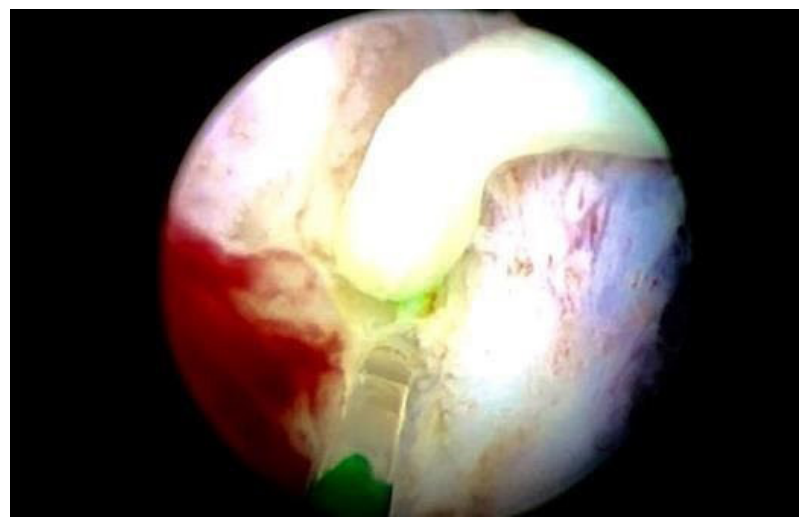

Figure 2. Holmium laser incision and purulent material drainage.

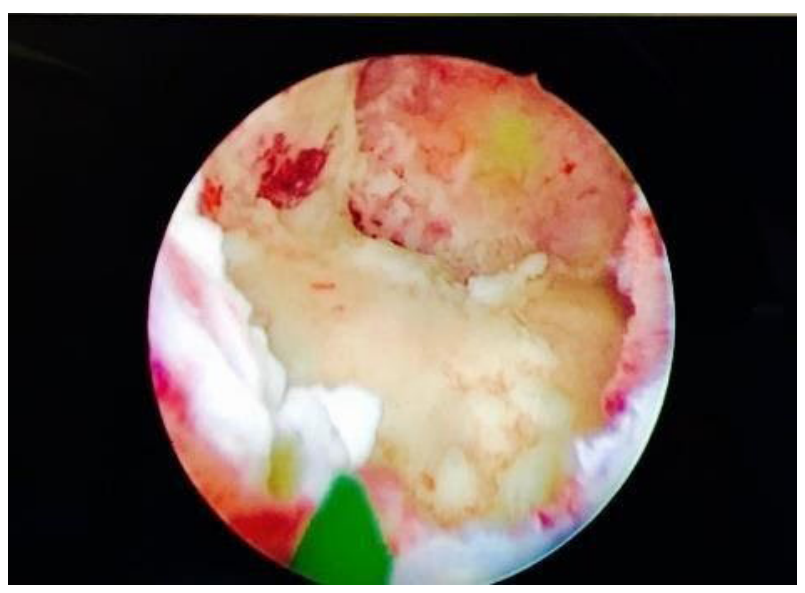

Figure 3. Abscess cavity opened.

infravesical obstruction, surgical instrumentation, immunosuppression, alcoholism, neurogenic bladder and advanced age. The growth in cultures of K. pneumoniae from purulent material of prostatic origin and the CSF suggests this as a possible origin of the prostatic infectious focus with subsequent infectious seeding through hematogenous route to the central nervous system.

In some cases, isolated antibiotic therapy can be effective for the resolution of the abscess, but in other cases, the evolution is torpid and can lead to an extremely critical situation if surgical drainage is not performed promptly. ${ }^{8}$

Minimally invasive drainage procedures include transrectal or perineal ultrasound- guided aspiration puncture with pig-tail placement and transurethral resection using monopolar or bipolar energy. However, though these techniques may be adequate in a patient with a single large abscess, they are not sufficient in multifocal abscess cavities. ${ }^{9}$

Holmium laser technology, which is currently used for the HoLEP technique (holmium laser enucleation of the prostate) in benign prostatic hyperplasia, as well as for the various endoscopic treatments of urolithiasis, can be useful in this type of drainage. By enabling controlled incisions of the prostatic tissue and working with saline solution to avoid the risk of transurethral resection 
syndrome, it allows better hemostasis for anticoagulated patients and patients with coagulopathy. ${ }^{4}$

The laser technology offers several further advantages, such as the ability to perform a simultaneous HoLEP for the elimination of the residual adenoma. This ensures the complete resection of the infected glandular prostate tissue. In published studies, up to a third of patients with prostate abscesses eventually require endoscopic treatment of the prostate due to associated benign prostatic hyperplasia (BPH). ${ }^{10,11}$

This case describes a minimally invasive technique as an alternative treatment to prostate abscesses. The holmium laser enabled resolution of the prostatic abscess and the benign prostatic hyperplasia in the same surgical procedure. However, there is still a lack of prospective randomized trials to provide further scientific evidence for the assessment of its true efficacy.

\section{Conclusion}

Prostatic abscesses require early diagnosis and treatment to avoid disseminated infections. The use of new technologies such as the holmium laser for the drainage of this pathology seems to be a valid option, providing an accurate and adequate hemostatic control in patients with coagulation disorders.

\section{Conflict of Interests}

The authors have no conflicts of interest to declare.

\section{Ethical Considerations}

Written informed consent was obtained from the patient for publication of this case report and any accompanying images.

\section{References}

1. Pierce JR Jr, Saeed Q, Davis WR. Prostatic abscess due to community-acquired methicillin-resistant Staphylococcus aureus. Am J Med Sci. 2008;335(2):154-6. doi: 10.1097/ MAJ.0b013e3180caac4b.
2. Weinberger M, Cytron S, Servadio C, Block C, Rosenfeld JB, Pitlik SD. Prostatic abscess in the antibiotic era. Rev Infect Dis. 1988;10(2):239-49. doi: 10.1093/clinids/10.2.239.

3. Liu KH, Lee HC, Chuang YC, Tu CA, Chang K, Lee NY et al. Prostatic abscess in southern Taiwan: another invasive infection caused predominantly by Klebsiella pneumoniae. J Microbiol Immunol Infect. 2003;36(1):31-6.

4. Lee CH, Ku JY, Park YJ, Lee JZ, Shin DG. Evaluation of holmium laser for transurethral deroofing of severe and multiloculated prostatic abscesses. Korean J Urol. 2015;56(2):150-6. doi: 10.4111/kju.2015.56.2.150.

5. Tiwari P, Pal DK, Tripathi A, Kumar S, Vijay M, Goel A, et al. Prostatic abscess: diagnosis and management in the modern antibiotic era. Saudi J Kidney Dis Transpl. 2011;22(2):298-301.

6. Liao CY, Yang YS, Yeh YC, Ben RJ, Lee CC, Tsai CC et al. Invasive liver abscess syndrome predisposed by Klebsiella pneumoniae related prostate abscess in a nondiabetic patient: a case report. BMC Res Notes. 2016; 9:395. doi: 10.1186/s13104-016-2188-y.

7. Ackerman AL, Parameshwar PS, Anger JT. Diagnosis and treatment of patients with prostatic abscess in the postantibiotic era. Int J Urol. 2018;25(2):103110. doi: 10.1111/ iju.13451.

8. Wen SC, Juan YS, Wang CJ, Chang K, Shih MC, Shen JT, et al. Emphysematous prostatic abscess: case series study and review. Int J Infect Dis. 2012;16(5): e344-9. doi: 10.1016/j. ijid.2012.01.002.

9. Goyal NK, Goel A, Sankhwar S, Dalela D. Transurethral resection of prostate abscess: is it different from conventional transurethral resection for benign prostatic hyperplasia? ISRN Urol. 2013; 2013:109505. doi: 10.1155/2013/109505.

10. Dajani AM, O’Flynn JD. Prostatic abscess. A report of 25 cases. Br J Urol. 1968;40(6):736-9. doi:10.1111/j.1464410x.1968.tb11875.x

11. Ludwig M, Schroeder-Printzen I, Schiefer HG, Weidner W. Diagnosis and therapeutic management of 18 patients with prostatic abscess. Urology. 1999;53(2):340-5. doi: 10.1016/ s0090-4295(98)00503-2. 